\title{
Una nueva asociación de dientes de vertebrados para la Formación Bajo de la Carpa (Santoniense, Cretácico Superior) en Rincón de los Sauces, Neuquén, Argentina
}

\author{
Leonardo S. FILIPPI ${ }^{*}$, Agustín G. MARTINELLI ${ }^{2}$ \& Alberto C. GARRIDO
}

\author{
${ }^{1}$ Museo Municipal Argentino Urquiza, Jujuy y Chaco s/n, (Q8319BFA), Rincón de los Sauces, Neuquén Patagonia, Argentina; \\ lsfilippi@yahoo.com.ar \\ ${ }^{2}$ Centro de Pesquisas Paleontológicas L.I. Price, CCCP/UFTM, BR-262, Km 784, Bairro Peirópolis, 38001-970, Uberaba, \\ MG, Brasil. / Departamento de Paleontologia e Estratigrafia, Instituto de Geociências, UFRGS, Porto Alegre, RS, Brasil; \\ agustin_martinelli@yahoo.com.ar \\ ${ }^{3}$ Museo Provincial de Ciencias Naturales "Prof. Dr. Juan Olsacher”. Dirección Provincial de Minería, Etcheluz y Ejército \\ Argentino, (Q8340AUD) Zapala, Neuquén, Argentina; albertocarlosgarrido@gmail.com \\ * Corresponding author
}

Filippi, L.S., Martinelli, A.G. \& Garrido, A.C. 2015. Una nueva asociación de dientes de vertebrados para la Formación Bajo de la Carpa (Santoniense, Cretácico Superior) en Rincón de los Sauces, Neuquén, Argentina. [A new vertebrate tooth assemblage from Bajo de la Carpa Formation (Santonian, Upper Cretaceous) of Rincón de los Sauces, Neuquén, Argentina]. Spanish Journal of Palaeontology, 30 (2), 223-238.

Manuscript received 29 November 2014

(C) Sociedad Española de Paleontología ISSN 2255-0550

Manuscript accepted 23 April 2015

\section{RESUMEN}

En el presente trabajo se da a conocer una nueva asociación de dientes de la Formación Bajo de la Carpa (Santoniense, Cretácico Superior) en un contexto paleoambiental diferente a las registradas en los alrededores de la ciudad de Neuquén y Paso Córdova (Patagonia, Argentina). Esta nueva asociación está integrada por dientes aislados de Dipnoi de las especies Ceratodus kaopen y Atlantoceratodus patagonicus; dientes de Mesoeucrocodylia, pertenecientes a Peirosauridae; dientes de terópodos Abelisauridae y dientes de saurópodos Titanosauria. Los especímenes de Mesoeucrocodylia, Abelisauridae y Titanosauria son consistentes con el registro fósil de la región, representado por la asociación de tetrápodos del Coloradoense para el intervalo Santoniense-Campaniense temprano. Sin embargo, el dipnoo A. patagonicus representa la primera ocurrencia para la Formación Bajo de la Carpa, siendo su registro el más antiguo para este taxón.

Palabras clave: Dipnoi, Mesoeucrocodylia, Abelisauria, Titanosauria, Cretácico Superior, Patagonia.

\begin{abstract}
In this contribution, a fossil tooth assemblage from the Bajo de la Carpa Formation (Santonian, Upper Cretaceous) in a different paleoenvironmental context from that recorded at the Neuquén city and Paso Córdova localities (Patagonia, Argentina), is here presented. It includes isolated teeth of Dipnoi, represented by the species Ceratodus kaopen and Atlantoceratodus patagonicus; mesoeucrocodylian isolated teeth referred to Peirosauridae; and isolated teeth of Abelisauridae theropods and Titanosauria sauropods. The Mesoeucrocodylia, Abelisauridae and Titanosauria taxa are consistent with the fossil record of the region, represented by the Coloradoan tetrapod assemblage of Santonian to early Campanian age. However, the lungfish $A$. patagonicus represents the first occurrence for the Bajo de la Carpa Formation, being this record the oldest one for this taxon.
\end{abstract}

Keywords: Dipnoi, Mesoeucrocodylia, Abelisauria, Titanosauria, Upper Cretaceous, Patagonia. 


\section{INTRODUCCIÓN}

El registro de asociaciones de vertebrados fósiles en el Cretácico Superior continental de la Cuenca Neuquina es abundante y permite una amplia apreciación de los componentes faunísticos para esa región. A pesar de que en muchas asociaciones faunísticas es difícil demostrar la contemporaneidad de sus componentes, ellas constituyen las principales herramientas para inferencias paleoecológicas de un determinado sitio paleontológico. En lo que respecta al registro del Cretácico de Nordpatagonia, diferentes asociaciones faunísticas fueron reconocidas en diversas unidades, tales como las de las formaciones Cerro Lisandro-Plottier (Localidad El Anfiteatro; Salgado et al., 2009), Portezuelo (Turoniense/Coniaciense) (Localidad Los Barreales; Calvo et al., 2007b), Bajo de la Carpa (Localidades de Paso Córdova y Ciudad de Neuquén; Bonaparte, 1991), Los Alamitos (Localidad Estancia Los Alamitos; Bonaparte et al., 1984; Bonaparte, 1987), Allen (Localidades Bajo de Santa Rosa y Bajo Trapalcó; Martinelli \& Forasiepi, 2004; Salgado et al., 2007; García \& Salgado, 2013), Loncoche (Localidades en el sur de Malargue; González Riga, 1999), entre otras. El abundante registro faunístico del Cretácico de Nordpatagonia ha permitido el establecimiento de asociaciones de tetrápodos dentro de una secuencia temporal (Leanza et al., 2004) con base en el Grupo Neuquén.

En este trabajo se presenta una asociación faunística de elementos dentales aislados, restringida a un nuevo sitio paleontológico en el área de Rincón de los Sauces (norte de la provincia de Neuquén) que, si bien procede de la ya bien conocida Formación Bajo de la Carpa, se ubican dentro de un contexto paleoambiental distinto al reconocido para las localidades de Bardas de Neuquén y Paso Córdova. Esta asociación ofrece nuevos datos para el estudio de la fauna de vertebrados de la Formación Bajo de la Carpa, Cretácico Superior del Grupo Neuquén.

Los primeros materiales, correspondientes a dientes de dinosaurios terópodos y cocodrilos, dados a conocer en este estudio fueron hallados en 2012 por un poblador de la localidad de Rincón de los Sauces, en el área del Yacimiento Narambuena, en las cercanías de una antigua locación petrolera (Fig. 1), y entregados al Museo Municipal Argentino Urquiza (MAU). Posteriores prospecciones en el sitio por una comisión del MAU dieron lugar al hallazgo de materiales adicionales. Los mismos provienen de un área no mayor a los 100 metros cuadrados en la cual se recuperaron nuevos dientes de dinosaurios terópodos y cocodrilos, además de dientes de saurópodos y el primer registro para la zona de dientes de peces, conjuntamente con fragmentos de placas de tortugas Chelidae y pequeños fragmentos de troncos fósiles.

\section{MATERIALES Y MÉTODOS}

Los materiales analizados en el presente estudio corresponden a dientes de peces, cocodrilos y dinosaurios terópodos y saurópodos, pertenecientes a la Colección Paleontológica del MAU, de la localidad de Rincón de los Sauces, Neuquén, Argentina. Para su estudio se utilizó una lupa binocular estereoscópica (Arcano ZTX 745L) con cámara de video (Arcano 1.3 Megapixeles), una cámara digital (Samsung PL120 14.2 Megapixeles) y para las mediciones un calibre vigesimal. Del total de dientes recuperados, se seleccionaron para estudio solo los mejor preservados y con caracteres diagnósticos.

Para la descripción de los dientes de dinosaurios terópodos la nomenclatura empleada es la siguiente: Cella y blood grooves (Buscalioni et al., 1997); CH: altura de la corona dental (Currie et al., 1990). FABL (Fore-aft basal length): Longitud mesio-distal de la base de la corona (Currie et al., 1990). CBW (Crown base width): Anchura latero-medial de la corona (Currie et al., 1990). CBR (CBW/ FABL): Índice de compresión lateral (Smith et al., 2005). CHR (CH/FABL): Índice de altura de la corona (Smith et al., 2005). $\mathbf{d m} / \mathbf{m m}$ : dentículos cada $5 \mathrm{~mm}$ en la carena mesial (Currie et al., 1990). dd/mm: dentículos cada $5 \mathrm{~mm}$ en la carena distal (Currie et al., 1990). DSDI (dm/ $/ \mathrm{mm} / \mathrm{dd} /$ $\mathrm{mm}$ ): Índice entre el número de dentículos entre la carena mesial y distal (Rauhut \& Werner, 1995). Dada la pobre conservación de la mayoría de los dientes no se han podido medir los dentículos en varias posiciones de las carenas.

Para el estudio de los dientes de saurópodos, la terminología empleada en la descripción ha sido tomada de Nowinski (1971), Ten Cate (1995), Upchurch \& Barrett (2000) y García \& Cerda (2010): labial (la cara externa), lingual (la cara interna), mesial (la cara rostral), distal (la cara caudal). Mientras que para documentar las medidas de los mismos, se ha modificado la terminología utilizada por Wilson (2005): CML (crown measure length): Medida de longitud de la corona. CBW (crown base width): Anchura latero-medial de la corona. LW (labiolingual width): Ancho labiolingual. WF (faceta de desgaste): Número y posición de las mismas.

Abreviaturas Institucionales: MACN-PV-RN, Museo Argentino de Ciencias Naturales "Bernardino Rivadavia" (PV, Paleontología de Vertebrados; RN, Colección Río Negro), Buenos Aires; MAU-Pv-N, Museo Municipal Argentino Urquiza, Paleontología de vertebrados, Narambuena; MUCPv, Museo de la Universidad del Comahue, Paleontología de Vertebrados; MML, Museo Municipal de Lamarque, Río Negro; MPCN-PV, Museo Patagónico de Ciencias Naturales, General Roca, Río Negro; MUCPv, Museo de la Universidad del Comahue, Paleontología de Vertebrados, Neuquén. 


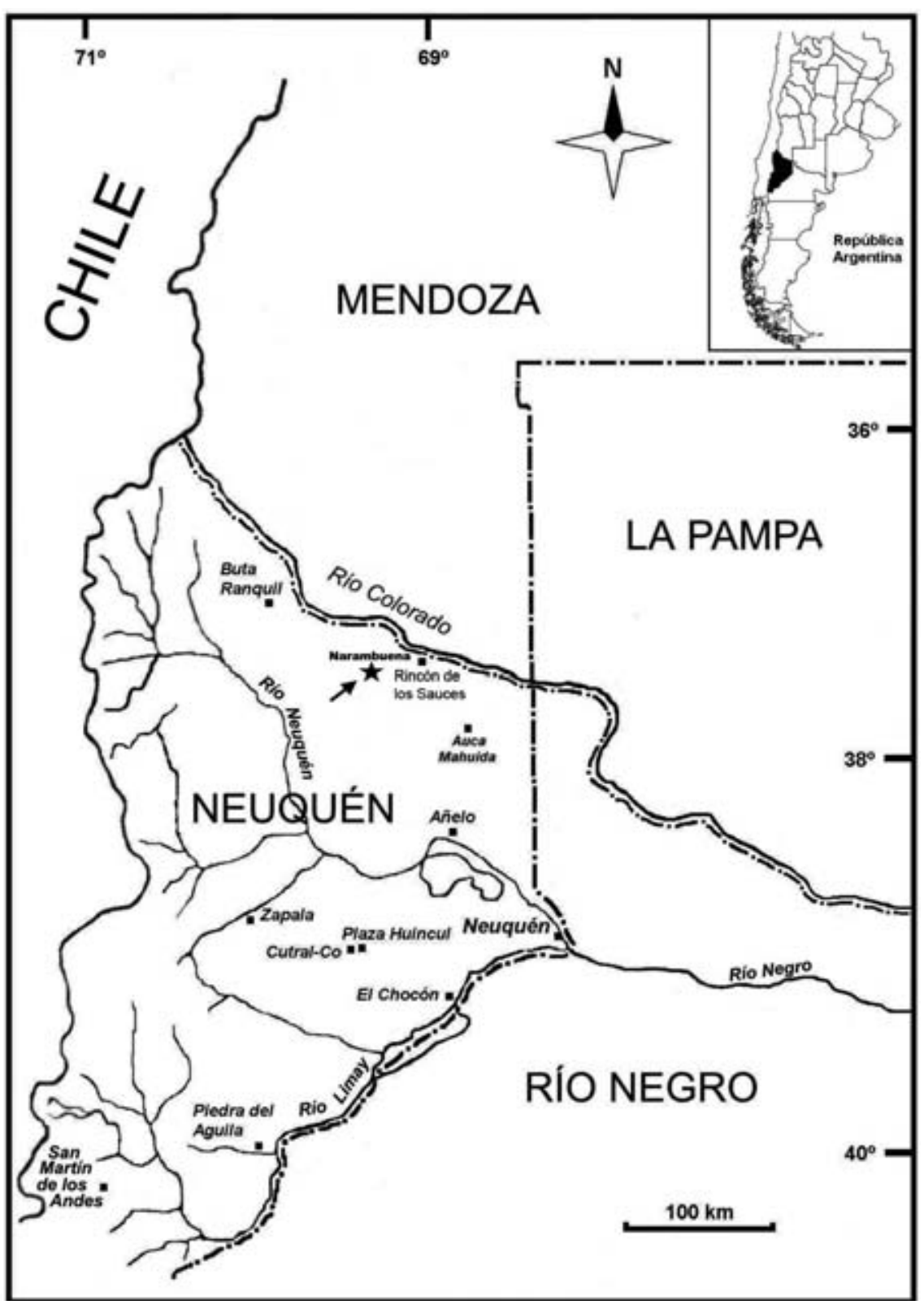

Figura 1. Mapa de ubicación del yacimiento Narambuena (estrella) donde fueron hallados los materiales.

\section{CONTEXTO GEOLÓGICO}

Los materiales de estudio proceden del yacimiento Narambuena $\left(37^{\circ} 25^{\prime} 21.57^{\prime}\right.$ 'S - 69 6 6 $52.87^{\prime}$ 'O), sitio ubicado aproximadamente a $17 \mathrm{~km}$ al suroeste de la ciudad de Rincón de los Sauces, provincia del Neuquén (Fig. 1). Los depósitos portadores corresponden a niveles superiores de la Formación Bajo de la Carpa, cuya edad ha sido situada por diversos autores dentro del Santoniense, Cretácico Superior (Legarreta \& Gulisano, 1989; Bonaparte, 1991; Hugo \& Leanza, 2001).

Esta unidad suele presentar a lo largo de la cuenca notables variaciones de espesor, tomando en la región de Rincón de los Sauces valores del orden de los 40 m de potencia (Cazau \& Uliana, 1973; Garrido, 2010).
Paleoambientalmente, se ha reconocido para la Formación Bajo de la Carpa en el sector central de la cuenca, el dominio de depósitos fluviales arenosos de baja sinuosidad, vinculados a canales de ríos excepcionalmente anchos y de baja profundidad (Garrido, 2010). Hacia el borde oriental de la cuenca, se menciona la presencia de depósitos de cauces entrelazados arenosos de escasa magnitud, alcanzando en algunos sectores un carácter efímero, asociados a planicies de inundación fangosas y a depósitos de dunas eólicas de moderado desarrollo (Calvo et al., 1997; Heredia \& Calvo, 1997, 2002; Caldwell \& Albino, 2001; Garrido \& Calvo, 2004; Sánchez et al., 2006).

Hasta el momento, la asociación faunística mejor documentada para la Formación Bajo de la Carpa, proviene de los depósitos eólicos de dunas e interdunas 
ubicados en los alrededores de la ciudad de Neuquén y Paso Córdova, sitios en los cuales han sido recuperados una variada fauna de cocodrilos, serpientes, restos óseos y huevos de aves enantiornites, junto a restos de dinosaurios terópodos alvarezsáuridos y abelisauroideos de pequeña talla (Woodward, 1896, 1901; Bonaparte, 1991; Alvarenga \& Bonaparte, 1992; Caldwell \& Albino, 2001; Schweitzer et al., 2002; Fiorelli \& Calvo, 2007; Martinelli \& Vera, 2007; Martinelli \& Pais, 2008; Fernández et al., 2013).

En relación con las litofacies, el nivel fosilífero del cual fueron recuperados los materiales de estudio, corresponde a un manto de arenisca cuarzolítica de granulometría mediana a fina, moderadamente seleccionada, conformando un horizonte tabular acuñado lateralmente. El mismo alcanza un espesor máximo de $15 \mathrm{~cm}$, presentándose intercalado en potentes y extensos depósitos de fangolitas rojas macizas. Internamente, el nivel portador psamítico presenta el desarrollo de estratificación horizontal, acompañada en ocasiones por una tenue estratificación ondulítica. Su base exhibe un contacto neto, ligeramente erosivo, en cuyos primeros centímetros se distribuyen granos de arena gruesa junto a abundantes intraclastos pelíticos. Es precisamente en la base de este horizonte donde se disponen y concentran los elementos colectados, sin orden aparente y con diverso grado de preservación, sugiriendo una concentración bajo la forma de depósitos residuales asociados a depósitos de desbordes sobre una planicie de inundación fangosa (Miall, 1996).

\section{PALEONTOlogía SiSTEMÁtica}

Subclase DiPNoI Muller, 1845

Orden Ceratodontiformes Berg, 1940

Género Ceratodus Agassiz, 1837

Ceratodus kaopen Apesteguía, Agnolín \& Claeson, 2007

Holotipo. MPCN-PV 1-1, placa dental inferior izquierda completa.

Procedencia del holotipo. Formación Bajo de la Carpa, localidad Paso Córdova, Río Negro, Argentina (Apesteguía et al., 2007).

Material referido. MAU-Pv-N 478/3, placa dental superior izquierda (Fig. 2a); MAU-Pv-N-536/2, placa dental superior derecha (Fig. 2b); MAU-Pv-N-478/1, placa dental superior izquierda (Fig. 2c); MAU-Pv-N-536/1, place inferior derecha (Fig. 2d); MAU-Pv-N-536/3, placa inferior derecha parcial (Fig. 2e).
Descripción. Los especímenes referidos a C. kaopen poseen una morfología similar entre ellos, con pequeñas diferencias en tamaño. Las placas superiores son más anchas transversalmente y más cortas mesiodistalmente, que las placas consideradas como inferiores. Todas las placas poseen la cuarta cresta baja y con una proyección distal que da una conformación cuadrangular a la región caudal de la placa, como es característico de la especie (Apesteguía et al., 2007). Esta característica produce un área de oclusión extensa y casi plana en esa región de la placa dental. Otras características de estas placas incluyen: la primera cresta de mayor tamaño, con un borde agudo y las crestas segunda y tercera sub-iguales en tamaño. Generalmente, la cuarta cresta, además de ser la menor de todas, se encuentra próxima a la tercera cresta. Las hendiduras son más pronunciadas en la porción mesial del diente y se torna casi superficial entre la tercera y cuarta cresta. Las crestas son mayormente rectas, siendo la primera suavemente curva. La superficie oclusal presenta marcas de erosión en algunos especímenes y se observa una ligera ornamentación formada por pequeños huecos.

Comentarios. El holotipo y materiales referidos de C. kaopen provienen de la Formación Bajo de la Carpa (Apesteguía et al., 2007). Asimismo, los numerosos materiales encontrados en Rincón de los Sauces indican una predominancia de esta especie sobre otros dipnoos (e.g., Atlantoceratodus patagonicus).

Género Atlantoceratodus Cione, Gouiric, Goin \& Poiré, 2007

\section{Atlantoceratodus patagonicus Agnolín, 2010}

Holotipo. MML 196, placa dental superior derecha (Agnolín, 2010: fig. 3a-b).

Procedencia del holotipo. Trapal Có, Bajo de Los Menucos, provincia de Río Negro, Argentina, Formación Allen (Campaniense Superior - Maastrichtiense, Cretácico Superior; Martinelli \& Forasiepi, 2004).

Material referido. MAU-Pv-N 478/2, placa dental superior izquierda (Fig. 2f).

Descripción. Un solo espécimen de esta especie fue encontrado en la localidad en estudio. Consiste en una placa dental superior izquierda con cinco crestas, con la primera cresta parcialmente rota al nivel de su base. A pesar de no estar disponible esta cresta, la sección rota sugiere que no era desarrollada como en las placas descritas anteriormente de Ceratodus kaopen (Figs 2a-2e). Asimismo, la segunda cresta posee un gran desarrollo, indicando que la primera cresta sería menor, como es autapomórfico para A. patagonicus (Agnolín, 2010). Las 


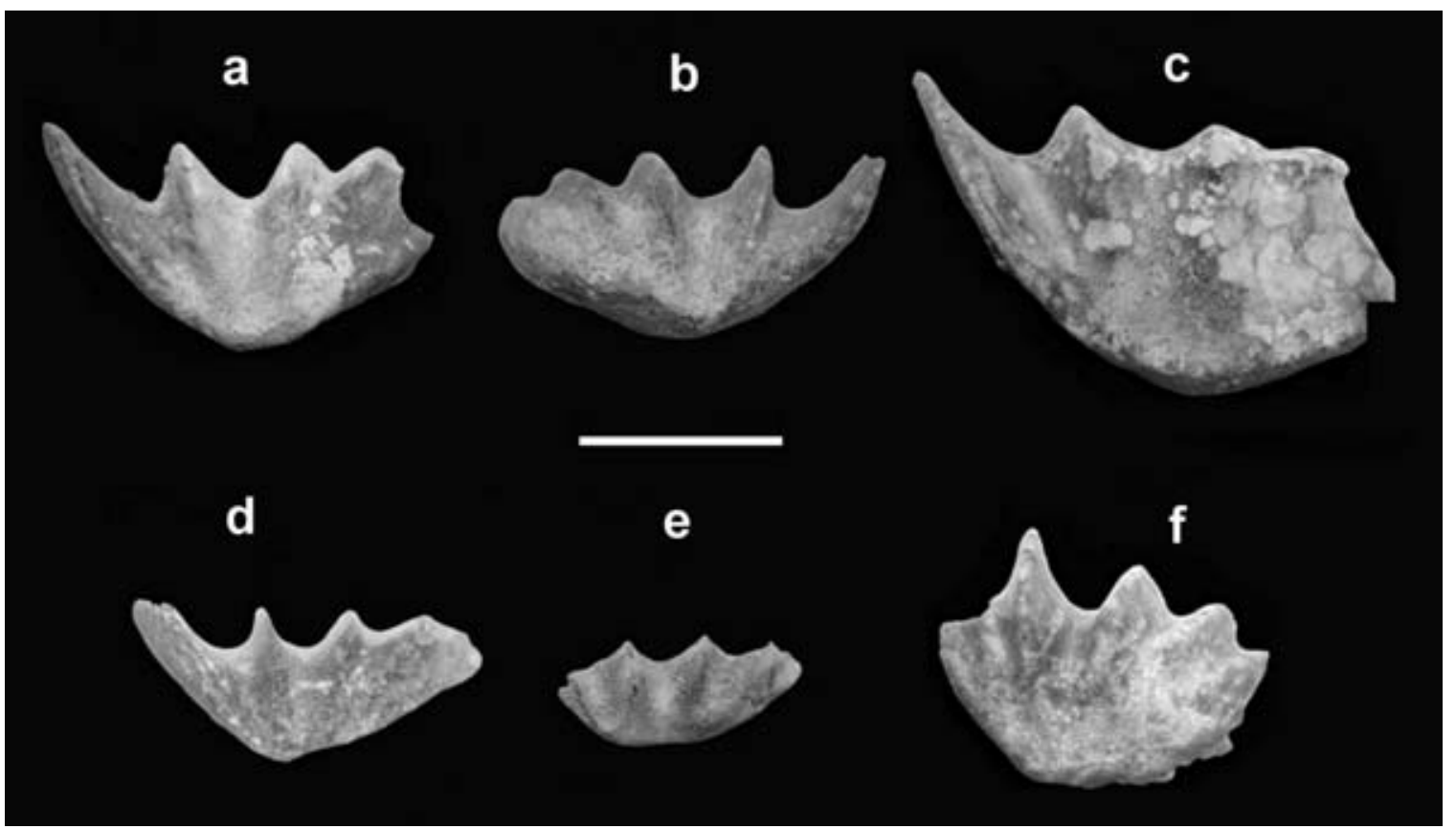

Figura 2. Placas dentales de Dipnoi, Ceratodus kaopen a-e) y Atlantoceratodus patagonicus f). (a) Placa dental superior izquierda (MAU-Pv-N 478/3). (b) Placa dental superior derecha (MAU-Pv-N-536/2). (c) Placa dental superior izquierda (MAU-Pv-N-478/1). (d) Placa dental inferior derecha (MAU-Pv-N-536/1). (e) Placa dental inferior derecha parcial (MAU-Pv-N-536/3). (f) Placa dental superior izquierda (MAUPv-N 478/2). Escala gráfica: $1 \mathrm{~cm}$.

crestas son de menor extensión que la plataforma oclusal. Las crestas descienden en tamaño posteriormente y la separación entre la segunda y tercera cresta es mayor que en las restantes, como en $A$. patagonicus. Las crestas son agudas y delgadas, separadas por surcos relativamente profundos. Las últimas dos crestas son sub-iguales, se posicionan próximas entre sí y se proyectan con la misma orientación, como es característico del género Atlantoceratodus (Agnolín, 2010). Este espécimen (MAUPv-N 478/2) posee la misma morfología que el holotipo descrito por Agnolín (2010: fig. 3A) proveniente de la Formación Allen, provincia de Río Negro.

Comentarios. Varias placas superiores e inferiores de A. patagonicus fueron colectadas en las localidades de Trapal Có y Bajo de Santa Rosa en rocas de la Formación Allen y la localidad Estancia Los Alamitos, en rocas de la Formación Los Alamitos (véase Agnolín, 2010). El registro aquí descrito indicaría la presencia de esta especie en estratos más antiguos, extendiendo el biocrón de $A$. patagonicus al Santoniense y su distribución geográfica al norte de la provincia de Neuquén.

Superorden Crocodylomorpha Hay, 1930 (sensu Walker, 1970)

Orden Crocodyliformes Hay, 1930 (sensu Benton \& Clark, 1988)
Suborden Mesoeucrocodylia Whetstone \& Whybrow, 1983

Mesoeucrocodylia indet.

Material referido. Dientes MAU-Pv-N-488/1-2, 489/1, 491, 493/1, 494/2, 495/1-2 у 5, 497, 524 у 526, corresponden a dientes aislados (Figs 3a-3s).

Comentarios. Debido a la variedad morfológica que presentan los dientes aquí descritos, hemos considerado separarlos en cuatro morfotipos diferentes: Morfotipo 1, caniniformes de gran tamaño, de sección subcircular; Morfotipo 2, caniniformes de mediano a pequeño tamaño, de sección oval; Morfotipo 3, molariformes de corona baja y mediano tamaño, de sección oval; y Morfotipo 4 , molariformes de corona baja y pequeño tamaño, de sección oval.

Morfotipo 1. MAU-Pv-N-491 (Fig. 3a), MAU$\mathrm{Pv}-\mathrm{N}-489 / 1$ (Figs 3b-3c), MAU-Pv-N-497 (Figs 3d3e), MAU-Pv-N-526 (Figs 3f-3g) y MAU-Pv-N-524 (Figs 3h-3i).

Descripción. Coronas altas de tipo caniniformes, de mayor tamaño (CH: 22 a $14 \mathrm{~mm}$, medidas estimadas debido a que la mayoría de la piezas se encuentran incompletas), de sección transversal subcircular, labiolingualmente 

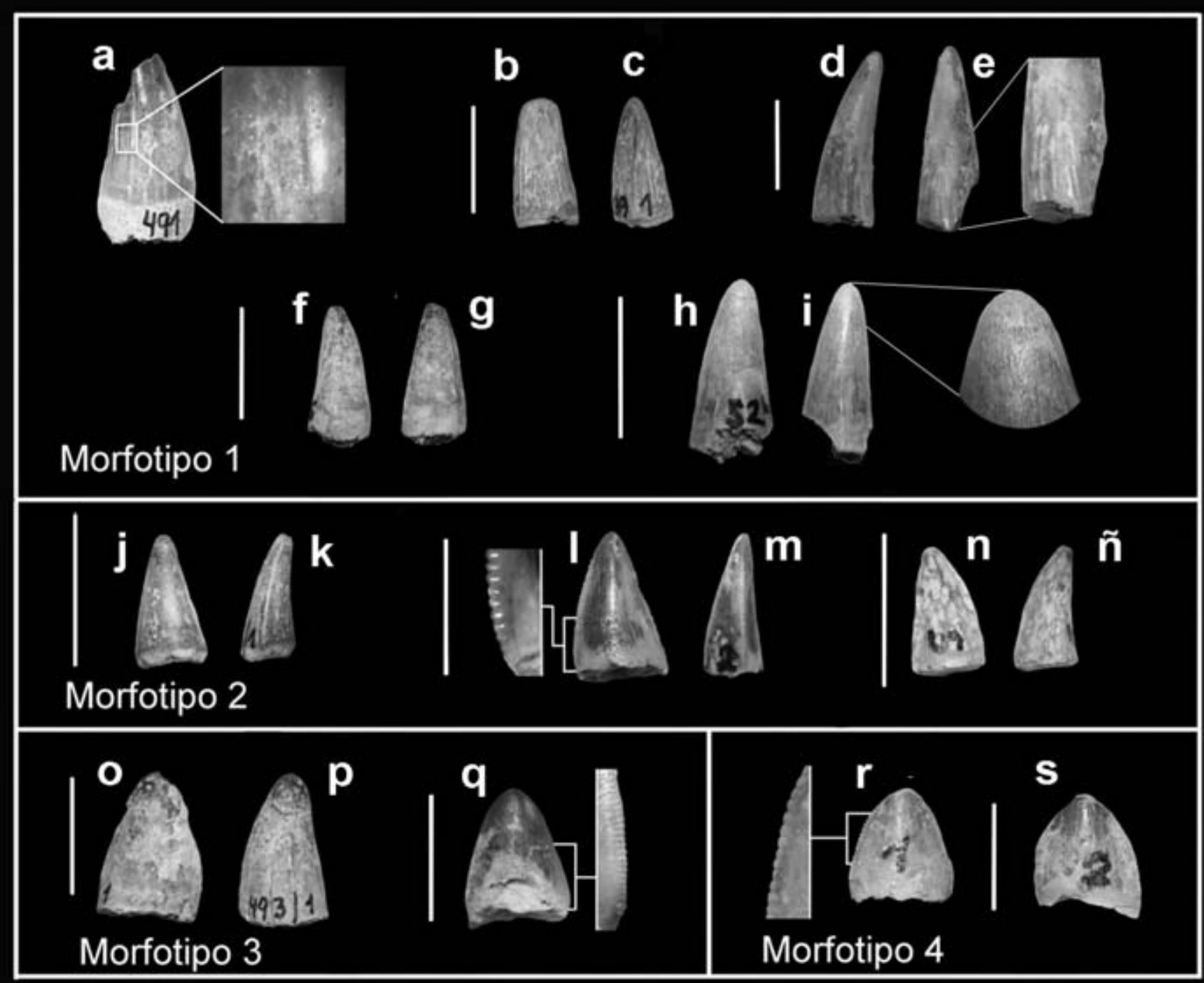

Figura 3. Dientes de Mesoeucrocodylia indet. a-s). Morfotipo 1. (a) Diente incompleto y detalle de dentículos sobre borde lateral cerca de la carena (MAU-Pv-N-491). b-c) Diente en vista lingual (b) y lateral (c) (MAU-Pv-N-489/1). d-e) Diente en vista lateral (d), lingual (e) y detalle de crestas o estrías longitudinales en la base de la corona (MAU-Pv-N-497). f-g) Diente en vista lateral (f) y labial (g) (MAU-Pv-N-526). h-i) Diente en vista labial (h) y lateral (i) con detalle de rugosidades en el esmalte (MAU-Pv-N-524). Morfotipo 2. j-k) Diente en vista lingual (j) y lateral (k) (MAU-Pv-N-495/1). l-m) Diente en vista lingual, con detalle de los dentículos (l), y vista lateral (m) (MAU-Pv-N-495/2). n-ñ) Diente en vista lingual (n) y lateral (ñ) (MAU-Pv-N-495/5). Morfotipo 3. o-p) Diente en vista labial (o) y lateral (p) (MAU-Pv-N-493/1). q) Diente en vista labial, com detalle de los dentículos (MAU-Pv-N-494/2). Morfotipo 4. r-s) Diente en vista labial, con detalle de los dentículos (MAU-Pv-N-488/1). s) Diente en vista labial (MAU-Pv-N-488/2). Escala gráfica: (a-q) $1 \mathrm{~cm},(\mathrm{r}-\mathrm{s}) 0.5 \mathrm{~cm}$.

recurvadas, con la cara labial convexa y la cara lingual cóncava. Ambas caras de la corona se encuentran separadas por carenas mesial y distal bien desarrolladas provistas de pequeños dentículos (dd/mm: 20, medida estimada tomada en el ejemplar MAU-Pv-N-491). En la mayoría de estas coronas se observan crestas o estrías longitudinales que, en algunos dientes van desde la base hasta el ápice en ambas caras y en otros solo en el tercio inferior de la corona. En el ejemplar MAU-Pv-N-491 se pueden observar en una de estas estrías o crestas la presencia de algunos dentículos aislados. En algunos dientes (e.g., MAU-Pv-N-489/1) se observa desgaste apical uniforme, sin presencia de facetas direccionadas.

Morfotipo 2. MAU-Pv-N-495/1 (Figs 3j-3k), MAUPv-N-495/2 (Figs 31-3m) y MAU-Pv-N-495/5 (Figs 3n-3ñ).

Descripción. Coronas de tipo caniniformes, de mediano a pequeño tamaño ( $\mathrm{CH}$ : 14 a $6 \mathrm{~mm}$ ), de sección oval, labiolingualmente recurvadas, con la cara labial convexa y la cara lingual cóncava a ligeramente cóncava. Ambas caras de la corona se encuentran separadas anterior y 
posteriormente por carenas medial y distalmente bien desarrolladas provistas de pequeños dentículos $(\mathrm{dm} / \mathrm{mm}: 15$ y dd/mm: 16, medidas tomadas en el ejemplar MAU$\mathrm{Pv}-\mathrm{N}-495 / 2$ ). El ejemplar MAU-Pv-N-495/5 presenta en sus dos caras estrías o crestas paralelas similares a las observadas en algunos de los especímenes del morfotipo 1 . En los dientes referidos a este morfotipo predomina el ápice agudo, con poco desgaste. Especialmente en el espécimen MAU-Pv-N-495/1 se observa una constricción en la base de la corona, donde comenzaría la raíz (no preservada).

Morfotipo 3. MAU-Pv-N-493/1 (Figs 3o-3p) y MAUPv-N-494/2 (Figs 3q).

Descripción. Coronas bajas de tipo molariformes, de mediano tamaño (CH: 14 a 9 mm), de sección oval a subcircular, levemente comprimidas labiolingualmente, con la cara labial y lingual levemente convexas, similares entre ellas. Ambas caras de la corona se encuentran separadas anterior y posteriormente por carenas mesial y distal bien desarrolladas provistas de pequeños dentículos muy pequeños (dd/mm: 34 , medida tomada en el ejemplar MAU-Pv-N-494/2). El ejemplar MAU-Pv-N-494/2 posee un esmalte ornamentado por rugosidades como ocurre en dientes correspondientes a los otros morfotipos descritos anteriormente. En el espécimen MAU-Pv-N-493/1 hay una constricción entre la corana y raíz. La raíz es extremadamente grande en comparación a la corona tanto en su desarrollo dorsoventral como en su sección transversal.

Morfotipo 4. MAU-Pv-N-488/1 (Fig. 3r) y MAUPv-N-488/2 (Fig. 3s).

Descripción. Coronas bajas de tipo molariformes, de pequeño tamaño $(\mathrm{CH}: 6 \mathrm{~mm})$, de sección oval, levemente comprimidas labiolingualmente, con la cara labial levemente convexa y la cara lingual ligeramente cóncava a plana. Ambas caras de la corona se encuentran separadas anterior y posteriormente por carenas mesial y distal provistas de pequeños dentículos $(\mathrm{dm} / \mathrm{mm}: 17$, medida tomada en el ejemplar 488/1). En el ejemplar MAU-Pv-N-488/2 se observa un surco poco profundo cerca de las carenas.

Discusión. Entre los dientes aquí descritos y asignados como Mesoeucrocodylia indet., se registran cuatro morfotipos diferenciados. Debido a la heterodoncia presente en numerosas especies de mesoeucrocodilios cretácicos e incluso en neosuquios fósiles y actuales (e.g., Prasad \& de Lapparent de Broin, 2002; Pol et al., 2014) los morfotipos descritos corresponderían a un único taxón. Los morfotipos no muestran claras especializaciones como aquellas observadas en diversas especies de notosuquios derivados, tales como Notosuchus y esfagesauridos (e.g., Lecuona \& Pol, 2008; Pol et al., 2014), ni presentan adaptaciones similares a las de sebecosuquios, tales como baurusuquidos (Riff \& Kellner, 2001; Carvalho et al., 2011). Por otro lado, los morfotipos se asemejan bastante al patrón presente en la serie maxilar y mandibular de peirosáuridos, que presentan heterodoncia (e.g., Gasparini et al., 1991; Carvalho et al., 2004, 2007; Martinelli et al., 2012). Particularmente, los ejemplares MAU$\mathrm{Pv}-\mathrm{N}-488 / 1-2$, designados como Morfotipo 4, poseen una gran similitud con el ejemplar MUCPv-411 descrito por Calvo \& Porfiri (2010) de la Formación Portezuelo, el cual es asignado por dichos autores como correspondiente a un Peirosauridae, probablemente a Lomasuchus palpebrosus (Gasparini et al., 1991). La similitud morfológica de los morfotipos 1 a 5 con los dientes maxilares y dentarios de Lomasuchus, Gasparinisuchus y Montealtosuchus indica que estos dientes corresponderían a un taxón de Peirosauridae.

Superorden Dinosauria Owen, 1842

Orden Saurischia Seeley, 1888

Suborden THEROPODA Marsh, 1881

Infraorden CERATOSAURIA Marsh, 1884

Superfamilia ABELISAUROIDEA Bonaparte, 1991

Abelisauroidea indet.

Material referido. Dientes aislados MAU-Pv- N-496/6 (Fig. 4a), MAU-Pv- N-498 (Fig. 4b), MAU-Pv- N-505 (Figs 4c-4d), MAU-Pv- N-507 (Fig. 4e), MAU-Pv- N-508 (Fig. 4f) y MAU-Pv- N-512 (Figs 4g-4h).

Descripción. Las coronas dentales son de pequeño a mediano tamaño (Tabla 1). Los dientes MAU-Pv-N-496/6, 498, 507 y 508 son comprimidos labiolingualmente y la sección de la base de la corona es de contorno ovalado. Por otro lado, los dientes MAU-Pv-N-505 y 512, poseen el lado labial más convexo y desarrollado que el lado lingual, y la sección de la base de la corona es de contorno subtriangular, en forma transversal de "D". Esta variación morfológica se debe a la posición que ocupa cada uno de los dientes; mientras los primeros (MAU-Pv-N-496/6, 498, 507 y 508) se ubican en una posición más distal, lateralmente sobre el dentario o el maxilar, los segundos (MAU-Pv-N-505 y 512) corresponden a elementos más anteriores, posiblemente del premaxilar. Los dientes MAUPv-N-496/6, 498, 507 y 508, se encuentran recurvados posteriormente, con el borde mesial convexo y el borde distal recto o levemente cóncavo. Por el contrario, y debido a la posición anatómica, los dientes MAU-Pv-N-505 y 512, poseen el borde mesial y distal recurvados hacia el ápice. Todos los dientes presentan el borde mesial y distal con 


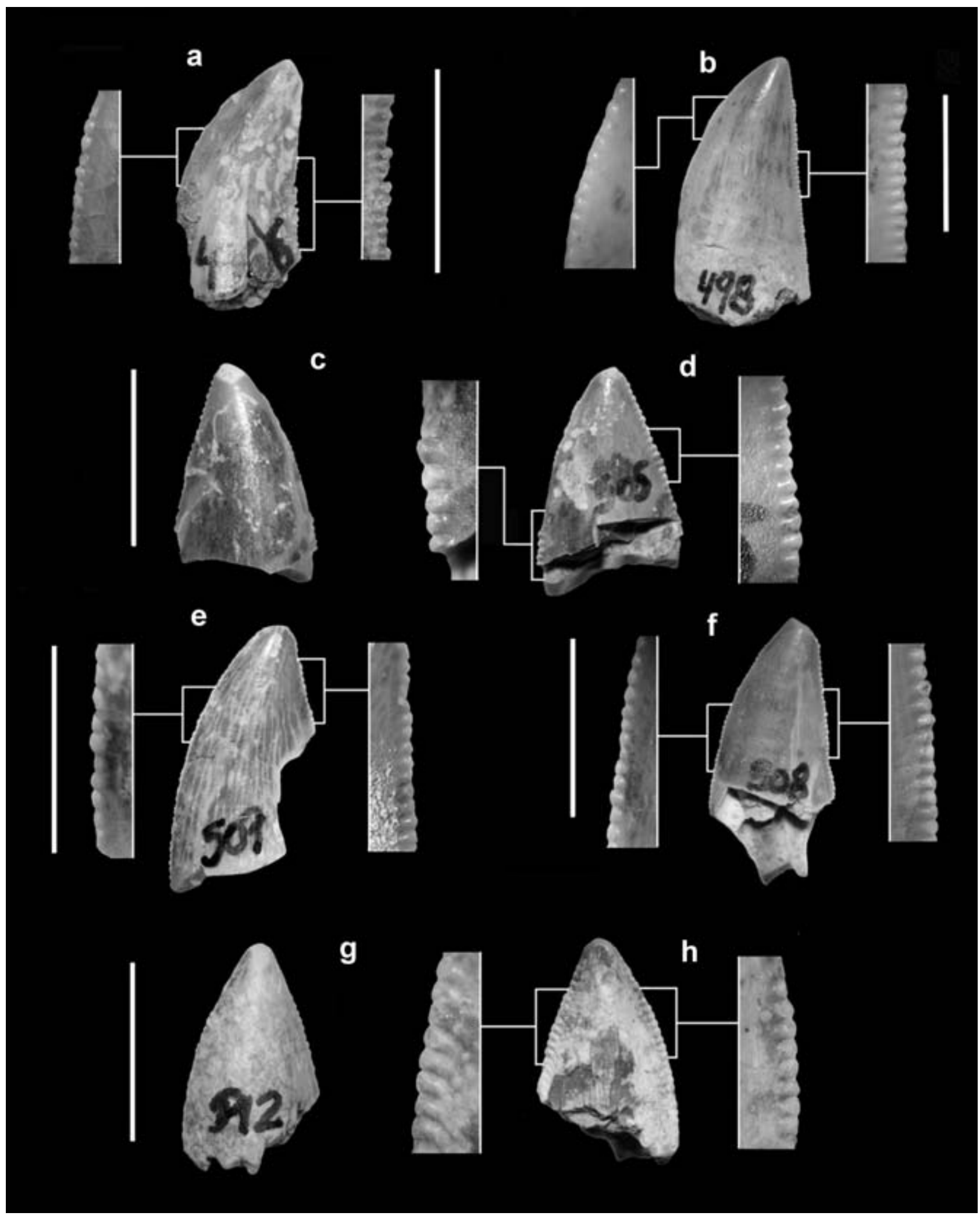

Figura 4. Dientes aislados de Terópoda Abelisauridae a-h). (a) Diente en vista labial, con detalle de los dentículos en la carenas mesial y distal (MAU-Pv- N-496/6). (b) Diente completo en vista labial, con detalle de los dentículos en la carenas mesial y distal (MAU-Pv- N-498). c-d) Diente en vista labial (c) y lingual con detalles de los dentículos en las carenas mesial y distal (d) (MAU-Pv- N-505). (e) Diente en vista labial, con detalle de los dentículos en la carenas mesial y distal (MAUPv- N-507). (f) Diente en vista labial, con detalle de los dentículos en la carenas mesial y distal (MAU-Pv- N-508). g-h) Diente em vista labial (g) y lingual con detalles de los dentículos en las carenas mesial y distal (h) (MAU-Pv- N-512). Escala gráfica: $1 \mathrm{~cm}$. 
Tabla 1. Medidas de los dientes de terópodos hallados en el sitio del yacimiento Narambuena (mm).

\begin{tabular}{lcccccccc}
\hline \multicolumn{1}{c}{$\mathbf{N}^{\mathbf{0}}$ Colección } & CH & FABL & CBW & CBR & CHR & $\mathbf{d m} / \mathbf{m m}$ & $\mathbf{d d} / \mathbf{m m}$ & DSDI \\
\hline MAU-PV-N-496/6 & $11,70^{*}$ & 5,5 & 2,2 & 0,40 & $2,13^{*}$ & 16 & 14 & 1,14 \\
MAU-PV-N-498 & 21,85 & 9,6 & 4,3 & 0,45 & 2,28 & 14 & 13 & 1,08 \\
MAU-PV-N-505 & $12,65^{*}$ & 8,6 & 4,7 & 0,55 & $1,47^{*}$ & 11 & 10 & 1,10 \\
MAU-PV-N-507 & $14,70^{*}$ & $?$ & 3,3 & $?$ & $?$ & 12 & 11 & 1,09 \\
MAU-PV-N-508 & $15,20^{*}$ & 7,2 & 3,5 & 0,48 & $2,11^{*}$ & 14 & 13 & 1,08 \\
MAU-PV-N-512 & $12,10^{*}$ & 8,9 & 4,3 & 0,48 & $1,36^{*}$ & 11 & 10 & 1,10 \\
\hline
\end{tabular}

*material incompleto

carenas provistas de dentículos, con una variación que va entre los 10 y los 16 dentículos cada $5 \mathrm{~mm}$. Los dentículos de la carena mesial son más pequeños o levemente más pequeños que los de la carena distal. Aunque algunos de los dentículos de las coronas se encuentran erosionados, se observa que poseen forma de cincel ("chisel-like") y se encuentran inclinados hacia el ápice. Entre los dentículos se desarrollan claramente cellae, no observándose hendiduras interdenticulares ("interdenticle slits") ni blood grooves. Los dientes MAU-Pv-N-498 y 508 presentan en uno de sus lados, suaves arrugas en el esmalte las que resultan difíciles de observar mediante fotografía, encontrándose distribuidas desde el borde de la carena distal hacia la mitad de la corona.

Discusión. Los dientes de terópodo estudiados presentan coronas con una fuerte convexidad del margen mesial, mientras que el margen distal es recto a levemente cóncavo, condición presente en Abelisauria (Smith et al., 2005). El pobre desarrollo de la curvatura de las coronas los diferencia notablemente de la mayoría de los terópodos, especialmente de los dientes de Maniraptora (Gauthier, 1986), cuya curvatura es notablemente más marcada. Debido a esto, el pobre desarrollo de la curvatura de las coronas es considerado por Smith y otros autores (2005) como una potencial sinapomorfía de Abelisauridae, presente en Majungasaurus, Rugops, Kryptops, además de abelisaurios de la India, abelisaurios indeterminados de Egipto y Patagonia (Smith \& Lamanna, 2006). Las arrugas observadas en algunos dientes, MAU-Pv-N-498 y 508, documentadas en especímenes de Carcharodontosauridae (Sereno et al., 1996; Novas et al., 2005; Coria \& Currie, 2006; Sereno \& Brusatte, 2008), también son conocidas para los abelisaurios (Canale et al., 2007, 2009; Ezcurra, 2009), por lo que esta característica no es exclusivas de carcarodontosaúridos. Aún así, existen diferencias en la curvatura labiolingual de los dientes; mientras en los abelisaurios los dientes son generalmente rectos, en los carcarodontosaúridos, como Mapusaurus rosae (Coria \& Currie, 2006) y Giganotosaurus carolinii (Coria \& Salgado, 1995) en vista anterior o posterior, presentan una curvatura labiolingual que le confiere un aspecto en forma de "S".
La combinación de caracteres en los dientes estudiados nos permite asignar estos dientes a Abelisauroidea indet. Asimismo, esta asignación resulta también acorde al conocimiento que se dispone sobre la fauna de tetrápodos de la asociación del Subgrupo Río Colorado (formaciones Bajo de la Carpa y Anacleto) para el intervalo SantonienseCampaniense temprano (Leanza et al., 2004), en el cual se registra la presencia de terópodos abelisauridos, tales como Abelisaurus comahuensis (Bonaparte \& Novas, 1985) y Aucasaurus garridoi (Coria et al., 2002), ambos provenientes de la Formación Anacleto. Hasta el momento, los materiales correspondientes a formas de terópodos de mediano tamaño para la Formación Bajo de la Carpa, solo se conocen a partir de restos parciales, uno proveniente de la localidad La Invernada, provincia de Neuquén, el cual corresponde a una pelvis casi completa (Porfiri \& Calvo, 2006) y otro proveniente de Paso Córdoba, provincia de Río Negro integrado por una única caudal anterior (Ezcurra \& Méndez, 2009), así como materiales dentales procedentes de La Bonita, Río Negro (Gianechini et al., 2011). Recientes trabajos de campo en la localidad La Invernada (Filippi et al., en prep.) recuperaron una nueva forma de Abelisauridae de pequeño-mediano tamaño muy completo y bien preservado.

Comentarios. Es importante destacar que entre los dientes de terópodo hallados, uno de los ejemplares MAUPv-N 496/1 (Figs 5a-5b) no presenta dentículos en el borde mesial como en el distal, y la superficie de la corona se encuentra muy desgastada y cubierta de pequeños huecos (pits), diferenciándose notablemente respecto del estado de preservación del resto de los dientes hallados en el sitio. Esta alteración en la superficie del esmalte no es interpretada como una variación morfológica de índole taxonómica. Una posible hipótesis es que estos dientes fueron tragados durante la alimentación, produciendo alteraciones en la superficie del esmalte por acción de los ácidos gástricos, antes de ser expulsados del cuerpo (Argast et al., 1987), tal como lo muestran dientes de terópodos hallados en condiciones similares en la Formación Judith River en Alberta, Canadá (Currie et al., 1990). Otra posible hipótesis, es la acción de procesos biogenéticos (Acosta 


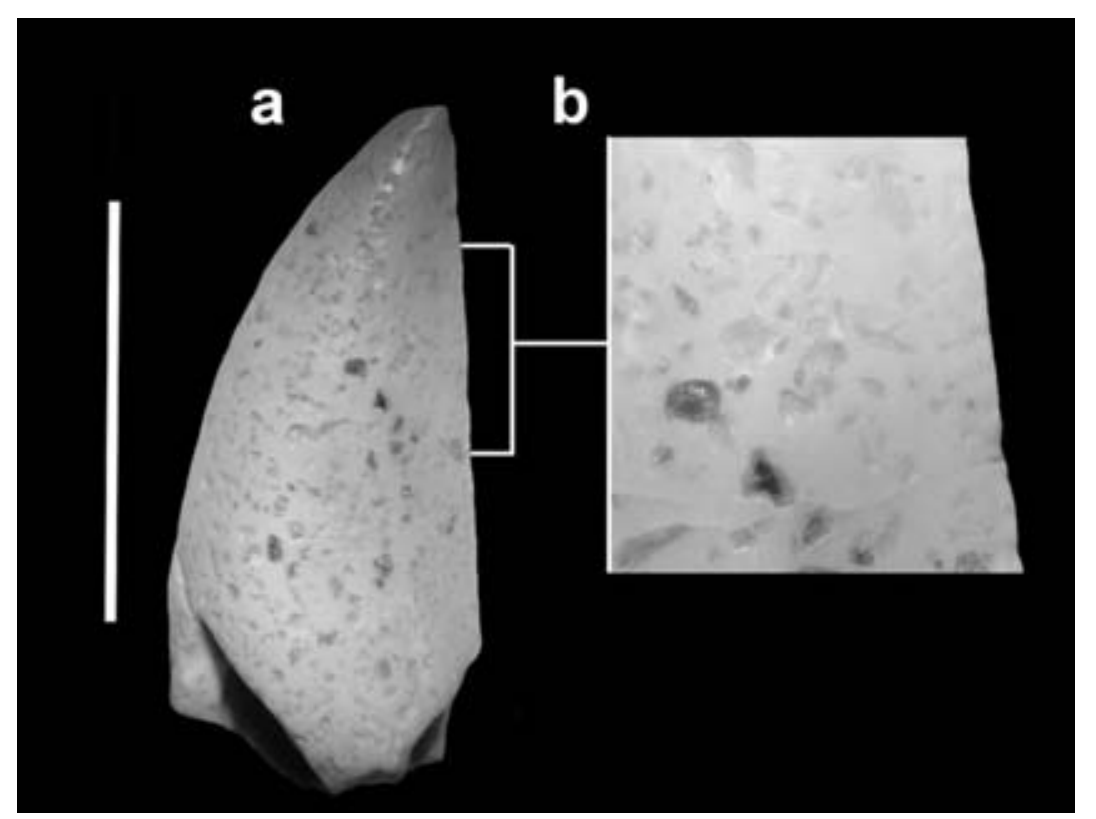

Figura 5. Diente de Terópoda indet. a-b). Diente en vista labial (a) y detalle del esmalte y borde de la carena distal sin dentículos (b) (MAU-Pv-N 496/1). Escala gráfica: $1 \mathrm{~cm}$.

Hospitaleche et al., 2011), aunque no queda demostrado en el presente trabajo. Aún así, la abrasión presente en estos dientes es un complejo fenómeno cuya modificación de su morfología original puede deberse a varios procesos diferentes (Argast et al., 1987).

\section{Superorden Dinosauria Owen, 1842 \\ Orden SAUrischia Seeley, 1888 \\ Suborden SAUROPODA Marsh, 1878 \\ Titanosauriformes Salgado, Coria \& Calvo, 1997}

TitAnosauria Bonaparte \& Coria, 1993

Titanosauria indet.

Material referido. Dientes aislados MAU-Pv-N-487/ 2 (Figs 6a-6c), MAU-Pv-N-487/1 (Figs 6d-6f), MAUPv-N-485 (Figs 6g-6i), MAU-Pv-N-482 (Figs 6j-61), MAU-Pv-N-483 (Figs 6m-6ñ), MAU-Pv-N-484(Figs 6o6q), MAU-Pv-N-481 (Figs 6r-6u) y MAU-Pv-N-479 (Figs $6 v-6 y)$.

Descripción. Con excepción de dos de los dientes (MAU-Pv-N-487/1-2), todos son cilíndricos, delgados, subcirculares en sección transversal, carecen de dentículos en la corona y varían en tamaño desde piezas grandes (MAU-Pv-N-481) hasta piezas más pequeñas (MAUPv-N-484) (Tabla 2). Los diferencia en la morfología y la diversidad de tamaños, corresponderían no solo a la variación de la talla de los individuos, sino también a su edad (García \& Cerda, 2010). La mayoría de los dientes son rectos, mientras que algunos de ellos (MAU-
Pv-N-481, 483 y 484) se encuentran levemente curvados en su tercio distal, interpretándose claramente en estos últimos, el lado labial, convexo y el lado lingual, cóncavo. Los dientes (MAU-Pv-N-487/1-2) mal preservados e incompletos, representados por los extremos de la corona, son de mayor tamaño y robustez. Poseen un lado lingual prácticamente plano delimitado por dos carenas longitudinales y un lado labial convexo. Ambos materiales poseen una ligera curvatura labiolingual en su tercio distal. Todos los materiales son interpretados como dientes funcionales, debido a la presencia de superficies de desgaste en el extremo de la corona. De acuerdo al modelo de clasificación propuesto por García \& Cerda (2010), sobre cantidad y posición de facetas de desgaste en dientes de saurópodos, se observa que los dientes aquí descritos poseen hasta dos facetas de desgaste. En la mayoría de los dientes que presentan una única faceta de desgaste, esta se ubica sobre el lado lingual (MAU-Pv-N-483,484, $485,487 / 2$ ) y solo en los ejemplares MAU-Pv-N-482 y $487 / 1$ en el borde lateral; mientras que en los dientes con dos facetas de desgaste (MAU-Pv-N-479 y 481), la mayor está ubicada sobre el lado lingual, mientras que la menor sobre el lado labial.

Discusión. Los dientes de saurópodos estudiados son asignados a Titanosauria debido a que corresponden a dientes en "forma de lápiz" (chisel-like; Calvo, 1994), caracterizados por ser en su mayoría delgados, subcirculares en sección transversal y carentes de dentículos en sus carenas. Si bien estos caracteres además de en Titanosauria, son compartidos por otro clado de Neosauropoda, los Diplodocoidea, ambos grupos difieren en la morfología y disposición de sus facetas de desgaste (García \& Cerda, 2010). 
Tabla 2. Medidas de los dientes de saurópodos hallados en el sitio del yacimiento Narambuena (mm).

\begin{tabular}{cccccc}
\hline \multicolumn{1}{c}{ N $^{\mathbf{0}}$ Colección } & CML & CBW & LW & WFN $^{\mathbf{c}}$ & WF posición \\
\hline MAU-PV-N-479 & $13,10^{*}$ & 4,1 & 3,6 & 2 & labial-lingual \\
MAU-PV-N-481 & $21,30^{*}$ & 4,2 & 3,5 & 2 & labial-lingual \\
MAU-PV-N-482 & $16,20^{*}$ & 3,8 & 3,1 & 1 & lateromedial \\
MAU-PV-N-483 & $12,40^{*}$ & 4,1 & 3,4 & 1 & lingual? \\
MAU-PV-N-484 & $10,50^{*}$ & 2,6 & 2,3 & 1 & lingual? \\
MAU-PV-N-485 & $11,70^{*}$ & 3,3 & 2,4 & 1 & lingual? \\
MAU-PV-N-487/1 & $19,50^{*}$ & $6,5^{*}$ & 6,2 & 1 & lingual? \\
MAU-PV-N-487/2 & $19,20^{*}$ & 6,8 & 5,5 & 1 & lingual? \\
\hline
\end{tabular}

*material incompleto

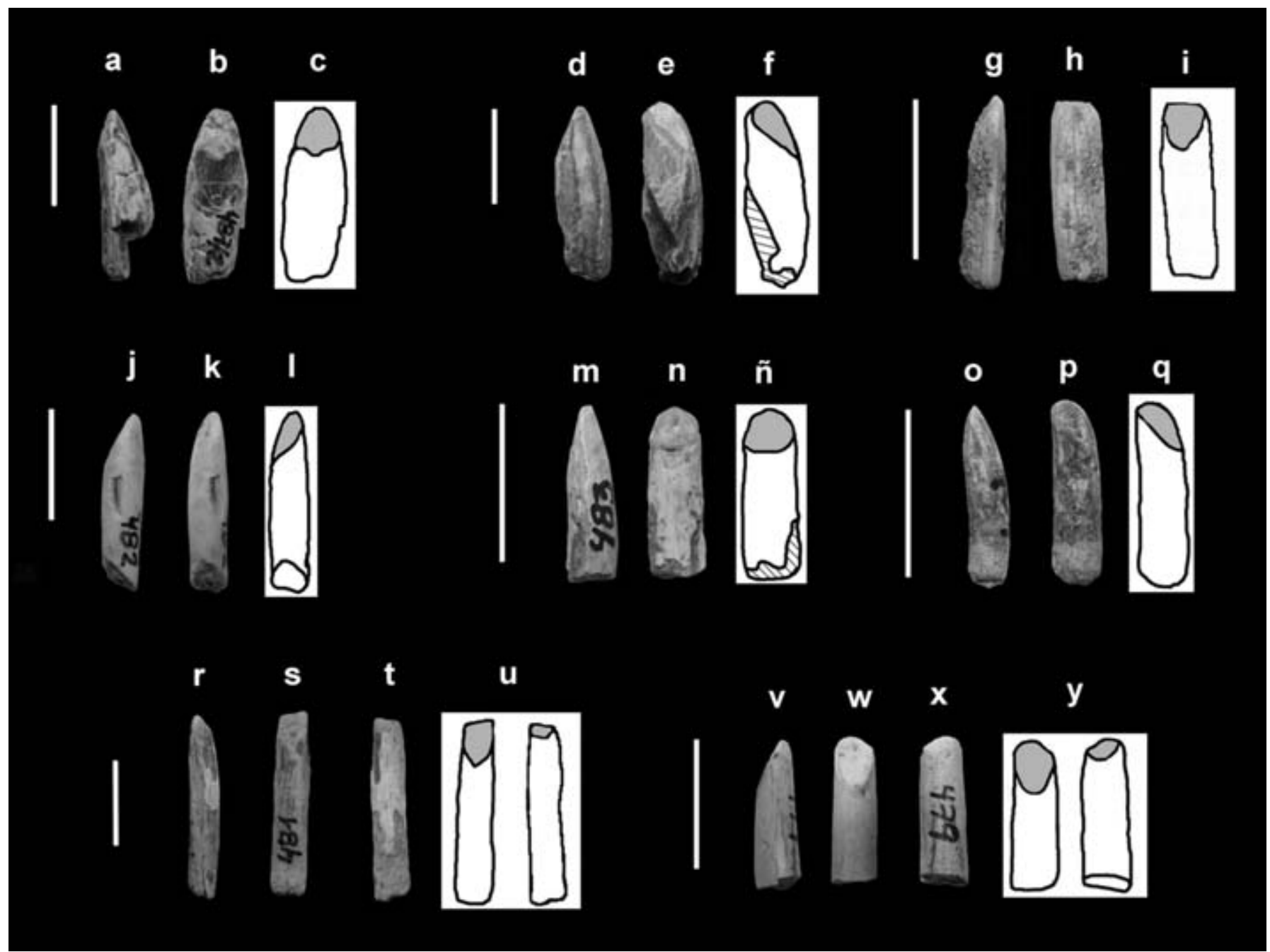

Figura 6. Dientes aislados de Saurópoda Titanosauria a-y). a-c) Diente en vista lateral (a), lingual (b) y detalle de la faceta de desgaste (c) (MAU-Pv-N-487/ 2). d-f) Diente en vista lateral (d), lingual (e) y detalle de la faceta de desgaste (f) (MAUPv-N-487/1). g-i) Diente en vista lateral (g), lingual (h) y detalle de la faceta de desgaste (i) (MAU-Pv-N-485). j-l) Diente en vista lateral (j), lingual (k) y detalle de la faceta de desgaste (l) (MAU-Pv-N-482). m-ñ) Diente en vista lateral (m), lingual (n) y detalle de la faceta de desgaste (ñ) (MAU-Pv-N-483). o-q) Diente en vista lateral (o), lingual (p) y detalle de la faceta de desgaste (q) (MAU-Pv-N-484). r-u) Diente en vista lateral (r), lingual (s), labial (t) y detalle de las facetas de desgaste (u) (MAU-Pv-N-481). v-y) Diente en vista lateral (v), lingual (w), labial (x) y detalle de las facetas de desgaste (y) (MAU-Pv-N-479). Escala gráfica: $1 \mathrm{~cm}$. 
Los dientes de tipo lápiz, son comunes en el registro de los titanosaurios del área de Rincón de los Sauces, como Rinconsaurus caudamirus (Calvo \& González Riga, 2003), Muyelensaurus pecheni (Calvo et al., 2007a), Pitekunsaurus macayai (Filippi \& Garrido, 2008) y Petrobrasaurus puestohernandezi (Filippi et al., 2011). Por su anatomía y la presencia de abundantes registros óseos de titanosaurios, los dientes hallados en este sitio son asignados como Titanosauria indet.

\section{CONCLUSIONES}

El presente trabajo permite dar a conocer una nueva asociación faunística de la Formación Bajo de la Carpa, ubicada en el área de Rincón de los Sauces, al norte de la provincia de Neuquén. A pesar de la condición fragmentaria de la mayoría de los especímenes descritos, por primera vez se registra a la especie Atlantoceratodus patagonicus (Dipnoi) para la Formación Bajo de la Carpa (Santoniense) en asociación con Ceratodus kaopen, citado anteriormente para esta misma unidad en la región de Paso Córdova (Provincia de Río Negro). El género Atlantoceratodus tiene una amplia distribución durante el Cretácico Superior (Cione et al., 2007; Agnolín, 2010); sin embardo, los registros de la especie A. patagonicus estaban restringidos al Campaniense Superior - Maastrichtiense de las formaciones Allen y Los Alamitos (véase Agnolín, 2010). De esta manera, la placa dental referida a $A$. patagonicus de la Formacion Bajo de la Carpa, de la región de Rincón de los Sauces, constituiría el registro más antiguo de la especie.

Los morfotipos de dientes de mesoeucrocodilios posiblemente representen un único taxón de Peirosauridae, un grupo bien documentado en el Grupo Neuquén, en las formaciones Portezuelo, Bajo de la Carpa y Anacleto (Gasparini et al., 1991; Calvo \& Porfiri, 2010; Martinelli et al., 2012).

En cuanto al registro de dientes de abelisáuridos y titanosaurios en este sitio de Rincón de los Sauces son consistentes con el registro fósil de la región y la edad para la asociación de tetrápodos Coloradoense propuesta por Leanza et al. (2004), la cual se caracteriza por la presencia de una gran diversidad de dinosaurios terópodos abelisaurios, con el registro de Abelisauridae basales (e.g., Bonaparte \& Novas, 1985) y carnotaurinos (e.g., Coria et al., 2002), saurópodos titanosáuridos, de los cuales se registra la primera aparición de saltasaurinos de pequeño porte, además de los grandes y gráciles eutitanosaurios basales (Leanza et al., 2004).

Hasta el momento la asociación faunística mejor documentada para la Formación Bajo de la Carpa, provenía de depósitos eólicos de dunas e interdunas ubicados en las bardas de la ciudad de Neuquén y Paso Córdova. Esta nueva asociación proveniente del Yacimiento Narambuena (Rincón de los Sauces, Neuquén) corresponde, por el contrario, a un paleoambiente notablemente diferente integrado por niveles de depósitos residuales asociados a depósitos de desbordes sobre una planicie de inundación fangosa.

\section{AGRADECIMIENTOS}

Deseamos expresar nuestro agradecimiento al Sr. Oscar Cruz por dar aviso del hallazgo del sitio al Museo, y entregar los primeros materiales motivos de este estudio, a los revisores Dr. R. A. Coria y al Dr. S. Apesteguía por sus comentarios y sugerencias que permitieron enriquecer el trabajo, al Lic. F. Agnolín por las discusiones sobre la problemática en la clasificación de los dientes de Dipnoi, y a la Municipalidad de Rincón de los Sauces por el apoyo logístico brindado durante los trabajos de campo.

\section{REFERENCIAS}

Acosta Hospitaleche, C., Márquez, G., Pérez, L., Rosato, V. \& Cione, A.L. 2011. Lichen bioerosion on fossil vertebrates from the Cenozoic of Patagonia and Antarctica. Ichnos, $18,1-8$.

Agnolin, F. 2010. A new species of the genus Atlantoceratodus (Dipnoiformes: Ceratodontoidei) from the Uppermost Cretaceous of Patagonia and a brief overview of fossil dipnoans from the Cretaceous and Paleogene of South America. Brazilian Geographical Journal: Geosciences and Humanities Research Medium, 1, 162-210.

Alvarenga, H. \& Bonaparte, J.F. 1992. A new flightless landbird from the Cretaceous of Patagonia. En: Papers in Avian Paleontology (ed. Campbell, K.E.). Natural History Museum of Los Angeles County, Science Series, 36, 51-64.

Apesteguía, S., Agnolín, F.L. \& Claeson, K. 2007. Review of Cretaceous dipnoans from Argentina (Sarcopterygii: Dipnoi) with descriptions of new species. Revista Museo Argentino de Ciencias Naturales, 9 (1), 27-40.

Argast, S., Farlow, J.O., Gabet, R.M. \& Brinkman, D.L. 1987. Transport-induced abrasion of fossil reptilian teeth: implications for the existence of Tertiary dinosaurs in the Hell Creek Formation, Montana. Geology, 15, 927-930.

Bonaparte, J.F. 1987. The Late Cretaceous fauna from Los Alamitos. Revista del Museo Argentino de Ciencias Naturales, Paleontología, 3, 103-178.

Bonaparte, J.F. 1991. Los vertebrados fósiles de la Formación Río Colorado, de la ciudad de Neuquén y cercanías, Cretácico superior, Argentina. Revista del Museo Argentino de Ciencias Naturales "Bernardino Rivadavia", Paleontología, 4 (3), 17-123.

Bonaparte, J.F. \& Novas, F.E. 1985. Abelisaurus comahuensis, n. g., n. sp., Carnosauria del Cretácico tardío de Patagonia. Ameghiniana, 21, 259-265. 
Bonaparte, J.F., Franchi, M.R., Powell, J.E. \& Sepúlveda, E.G. 1984. La Formación Los Alamitos (CampanianoMaastrichtiano) del sudeste de Río Negro, con descripción de Kritosaurus australis n. sp. (Hadrosauridae). Significado paleogeográfico de los vertebrados. Revista de la Asociación Geológica Argentina, 39, 284-299.

Buscalioni, A.D., Gasparini, Z., Pérez-Moreno, B.P. \& Sanz, J.L. 1997. Argentinean theropods: first morphological analysis on isolated teeth. En: I European Workshop on Vertebrate Palaeontology. Geological Society of Denmark, On Line Series 1 (www.2dgf.dk/ online/online01.htm).

Caldwell, M.W. \& Albino, A.M. 2001. Palaeoenvironment and palaeoecology of three Cretaceous snakes: Pachyophis, Pachlyrhachis, and Dinilysia. Acta Palaeontologica Polonica, 46, 1-86.

Calvo, J.O. 1994. Jaw mechanics in sauropod dinosaurs. Gaia, 10, 183-193.

Calvo, J.O., Engelland, S., Heredia, S. \& Salgado, L. 1997. First record of dinosaur eggshells (?SauropodaMegaloolithidae) from Neuquén, Patagonia, Argentina. Gaia, 14, 23-32.

Calvo, J.O. \& González-Riga, B.J. 2003. Rinconsaurus caudamirus gen. et sp. nov., a new titanosaurid (Dinosauria, Sauropoda) from the Late Cretaceous of Patagonia, Argentina. Revista Geológica de Chile, 30, 333-353.

Calvo, J.O., González-Riga, B.J. \& Porfiri, J.D. 2007a. A new titanosaur sauropod from the Late Cretaceous of Neuquén, Patagonia, Argentina. Arquivos do Museu Nacional, 65, 485-504.

Calvo, J.O. \& Porfiri, J.D. 2010. New material of peirosaurids from Neuquén, Patagonia: its age. Brazilian Geographical Journal: Geosciences and Humanities Research Medium, 1, 50-64.

Calvo, J.O., Porfiri, J.D., González Riga, B.J. \& Kellner, A.W.A. 2007b. A new Cretaceous terrestrial ecosystem from Gondwana with the description of a new sauropod dinosaur. Anais da Academia Brasileira de Ciências, 79, 529-541.

Canale, J.I., Agnolin, F.L., Ezcurra, M.D. \& Novas, F.E. 2007. Notas sobre el registro de dientes aislados de dinosaurios terópodos Cretácicos de América del Sur. Ameghiniana, 44 (4), 8R.

Canale, J.I., Scanferla, C.A., Agnolin, F.L. \& Novas, F.E. 2009. New carnivorous dinosaur from the Late Cretaceous of NW Patagonia and the evolution of abelisaurid theropods. Naturwissenschaften, 96, 409-414.

Carvalho, I.S., Ribeiro, L.C.B. \& Avilla, L.S. 2004. Uberabasuchus terrificus sp. nov., a new Crocodylomorpha from the Bauru Basin (Upper Cretaceous), Brazil. Gondwana Research, 7, 975-1002.

Carvalho, I.S., Vasconcellos, F.M. \& Tavares, S.A.S. 2007. Montealtosuchus arrudacamposi, a new peirosaurid crocodile (Mesoeucrocodylia) from the Late Cretaceous Adamantina Formation of Brazil. Zootaxa, 1607, 35-46.

Carvalho, I.S., Teixeira, V.P.A., Ferraz, M.L.F., Ribeiro, L.C.B., Martinelli, A.G., Neto, F.M., Sertich, J.J., Cunha, G.C., Cunha, I.C. \& Ferraz, P.F. 2011. Campinasuchus dinizi gen. et sp. nov., a new Late Cretaceous baurusuchid
(Crocodyliformes) from the Bauru Basin, Brazil. Zootaxa, 2871, 19-42.

Cazau, L.B. \& Uliana, M.A. 1973. El Cretácico superior continental de la Cuenca Neuquina. $5^{\circ}$ Congreso Geológico Argentino, Actas, 3, 131-163.

Cione, A.L., Gouiric, S., Goin, F. \& Poiré, D. 2007. Atlantoceratodus, a new genus of lungfish from the upper Cretaceous of South America and Africa. Revista del Museo de La Plata, 10, 1-12.

Coria, R.A. \& Currie, P.J. 2006. A new carcharodontosaurid (Dinosauria, Theropoda) from the Upper Cretaceous of Argentina. Geodiversitas, 28, 71-118.

Coria, R.A., Chiappe, L.M. \& Dingus, L. 2002. A new close relative of Carnotaurus sastrei Bonaparte 1985 (Theropoda: Abelisauridae) from the Late Cretaceous of Patagonia. Journal of Vertebrate Paleontology, 22, 460-465.

Coria, R.A. \& Salgado, L. 1995. A new giant carnivorous dinosaur from the Cretaceous of Patagonia. Nature, 377 , 224-226.

Currie, P.J., Rigby, J.K. \& Sloan, R.E. 1990. Theropod teeth from the Judith River Formation of Southern Alberta, Canada. En: Dinosaurs systematics. Approaches and Perspectives (eds Carpenter, K. \& Currie, P.J.). Cambridge University Press, Cambridge, 282-298.

Ezcurra, M.D. 2009. Theropod remains from the uppermost Cretaceous of Colombia and their implications for the palaeozoogeography of western Gondwana. Cretaceous Research, 30 (5), 1339-1344.

Ezcurra, M.D. \& Méndez, A.H. 2009. First report of a derived abelisaurid theropod from the Bajo de la Carpa Formation (Late Cretaceous), Patagonia, Argentina. Bulletin of Geosciences, 84 (3), 547-554.

Fernández, M.S., García, R.A., Fiorelli, L., Scolaro, A., Salvador, R.B., Cotaro, C.N., Kaiser G.W. \& Dyke, G.J. 2013. Large accumulation of avian eggs from the Late Cretaceous of Patagonia (Argentina) reveals a novel nesting strategy in Mesozoic birds. PlosOne, 8 (4), e61030.

Filippi, L.S. \& Garrido, A.C. 2008. Pitekunsaurus macayai gen. et sp. nov., nuevo titanosaurio (Saurischia, Sauropoda) del Cretácico Superior de la Cuenca Neuquina, Argentina. Ameghiniana, 45, 575-590.

Filippi, L.S., Canudo, J.I., Salgado, L.J., Garrido, A.C., García, R.A., Cerda, I.A. \& Otero, A. 2011. A new sauropod titanosaur from the Plottier Formation (Upper Cretaceous) of Patagonia (Argentina). Geologica Acta, 9 (1), 1-12.

Fiorelli, L. \& Calvo, J.O. 2007. The first "protosuchian" (Archosauria: Crocodyliformes) from the Cretaceous (Santonian) of Gondwana. Arquivos do Museu Nacional, 65 (4), 417-459.

Gasparini, Z., Chiappe, L.M \& Fernández, M.A. 1991. New Senomanian peirosaurid (Crocodylomorpha) from Argentina and synopsis American Cretaceous crocodilian. Journal of Vertebrate Palaeontology, 11 (3), 316-333.

García, R.A. \& Cerda, I.A. 2010. Dentición de titanosaurios (Dinosauria, Sauropoda) del Cretácico Superior de la provincia de Río Negro, Argentina: morfología, inserción y reemplazo. Ameghiniana, 47 (1), 45-60. 
García, R.A. \& Salgado, L. 2013. The titanosaur sauropods from the late Campanian-early Maastrichtian Allen Formation of Salitral Moreno, Río Negro, Argentina. Acta Palaeontologica Polonica, 58 (2), 269-284.

Garrido, A. 2010. Estratigrafía del Grupo Neuquén, Cretácico Superior de la Cuenca Neuquina (Argentina): nueva propuesta de ordenamiento litoestratigráfico. Revista del Museo Argentino de Ciencias Naturales "Bernardino Rivadavia”, n. s., 12, 121-177.

Garrido, A.C. \& Calvo, J.O. 2004. Reasignación litoestratigráfica de los niveles portadores de Megaloolithus patagonicus Calvo et al. (Dinosauria: Sauropoda) en las bardas de la ciudad de Neuquén. Ameghiniana, 41(4); 47R.

Gauthier, J.A. 1986. Saurischian monophyly and the origin of birds. Memoirs of the California Academy of Sciences, $8,1-55$.

Gianechini, F.A., Lio, G. \& Apesteguía, S. 2011. Isolated archosaurian teeth from "La Bonita" locality (Late Cretaceous, Santonian-Campanian), Río Negro Province, Argentina. Historia Natural, 3 (1), 5-16.

González Riga, B.J. 1999. Hallazgo de vertebrados fósiles en la Formación Loncoche, Cretácico Superior de la provincia de Mendoza, Argentina. Ameghiniana, 36, 401-410.

Heredia, S. \& Calvo, J.O. 1997. Sedimentitas eólicas en la Formación Río Colorado (Grupo Neuquén) y su relación con la fauna del Cretácico Superior. Ameghiniana, 34 (1), 120.

Heredia, S. \& Calvo, J.O. 2002. Estratigrafía de las bardas de la ciudad de Neuquén, Argentina. $\mathrm{XV}^{\circ}$ Congreso Geológico Argentino, Actas, 699-705.

Hugo, C.A. \& Leanza, H.A. 2001. Hoja Geológica 3069-IV General Roca (escala 1:250.000). Provincias de Río Negro y Neuquén. Instituto de Geología y Recursos Minerales. Boletín SEGEMAR, 308, 1-65.

Leanza, H.A., Apesteguía, S., Novas, F.E. \& de la Fuente, M.S. 2004. Cretaceous terrestrial beds from the Neuquén basin (Argentina) and their tetrapod assemblages. Cretaceous Research, 25 (1), 1-96.

Lecuona, A. \& Pol, D. 2008. Tooth morphology of Notosuchus terrestris (Notosuchia: Mesoeucrocodylia): New evidence and implications. Comptes Rendus Palevol, 7 (7), 407-417.

Legarreta, L. \& Gulisano, C.A. 1989. Análisis estratigráfico secuencial de la Cuenca Neuquina (Triásico superior Terciario inferior). En: Cuencas Sedimentarias Argentinas (eds. Chebli, G. \& Spalletti, L.), $10^{\circ}$ Congreso Geológico Argentino. Serie Correlación Geológica, 6, 221-243.

Martinelli, A.G. \& Forasiepi, A.M. 2004. Late Cretaceous vertebrates from Bajo de Santa Rosa (Allen Formation), Río Negro Province, Argentina, with the description of a new sauropod dinosaur (Titanosauridae). Revista del Museo Argentino de Ciencias Naturales "Bernardino Rivadavia”, n.s., 6 (2), 257-305.

Martinelli, A.G. \& Vera, E.I. 2007. Achillesaurus manazzonei, a new alvarezsaurid theropod (Dinosauria) from the Late Cretaceous Bajo de la Carpa Formation, Río Negro Province, Argentina. Zootaxa, 1582, 1-17.
Martinelli, A.G. \& Pais, D.F. 2008. A new baurusuchid crocodyliform (Archosauria) from the Late Cretaceous of Patagonia (Argentina). Comptes Rendus Paleovol, 7 (6), 371-381.

Martinelli, A.G., Sertich J.J.W., Garrido A.C. \& Praderio, A.M. 2012. A new peirosaurid from the Late Cretaceous of Argentina: implications for specimens referred to Peirosaurus torminni Price (Crocodyliformes: Peirosauridae). Cretaceous Research, 37, 191-200.

Miall, A.D. 1996. The geology of fluvial deposits. Sedimentary facies, basin analysis, and petroleum geology. Springer, New York.

Novas, F.E., de Valais, S., Vickers-Rich, P. \& Rich, T. 2005. A large Cretaceous theropod from Patagonia, Argentina, and the evolution of carcharodontosaurids. Naturwissenschaften, 92, 226-230.

Nowinski, A. 1971. Nemegtosaurus mongoliensis n. gen., n. sp. (Sauropoda) from the Uppermost Cretaceous of Mongolia. Palaeontologia Polonica, 25, 57-81.

Pol, D., Nascimento, P.M., Carvalho, A.B., Riccomini, C., PiresDomingues, R.A. \& Zaher, H. 2014. A new notosuchian from the Late Cretaceous of Brazil and the phylogeny of advanced Notosuchians. PlosOne, 9 (4), e93105.

Porfiri, J.D. \& Calvo, J.O. 2006. A new record of Carnotaurinae (Theropoda Abelisauridae) from the Upper Cretaceous of Neuquén, Patagonia. Journal of Vertebrate Paleontology, Abstracts, 26 (3), 111.

Prasad, G.V.R. \& de Lapparent de Broin, F. 2002. Late Cretaceous crocodile remains from Naskal (India): comparisons and biogeographic affinities. Annales de Paléontologie, 88, 19-71.

Rauhut, O.W.M. \& Werner, C. 1995. First record of the Family Dromaeosauridae (Dinosauria: Theropoda) in the Cretaceous of Gondwana (Wadu Milk Formation, northern Sudan). Paläontologische Zeitschrift, 69, 475-489.

Riff, D. \& Kellner, A.W.A. 2001. On the dentition of Baurusuchus pachecoi Price (Crocodyliformes, Metasuchia) from the Upper Cretaceous of Brazil. Boletim do Museu Nacional, n.s., 59, 1-15.

Salgado, L., Coria, R.A., Magalhães Ribeiro, C.M., Garrido, A., Rogers, R., Simon, M. E., Arcucci, A.B., Rogers, K.C., Carabajal, A.P., Apesteguía, S., Fernandez, M., García, R.A. \& Talevi, M. 2007. Upper Cretaceous dinosaur nesting sites of Río Negro (Salitral Ojo de Agua and Salinas de Trapalco-Salitral de Santa Rosa), northern Patagonia, Argentina. Cretaceous Research, 28 (3), 392-404.

Salgado, L., Canudo, J.I., Garrido, A.C., Ruiz-Omeñaca, J.I., García, R.A., de la Fuente, M.S., Barco, J.L. \& Bollati, R. 2009. Upper Cretaceous vertebrates from El Anfiteatro area, Rio Negro, Patagonia, Argentina. Cretaceous Research, 30, 767-784.

Sánchez, M.L., Gómez, M.J. \& Heredia, S. 2006. Sedimentología y paleoambientes del Subgrupo Río Colorado (Cretácico Superior), Grupo Neuquén, en las bardas de la ciudad de Neuquén y alrededores. Revista de la Asociación Geológica Argentina, 61 (2), 236-255.

Schweitzer, M.H., Jackson, F.D., Chiappe, L.M, Schmitt, J.G., Calvo, J.O. \& Rubilar, D.E. 2002. Late Cretaceous avian 
eggs with embryos from Argentina. Journal of Vertebrate Paleontology, 22 (1), 191-195.

Sereno, P.C. \& Brusatte, S.L. 2008. Basal abelisaurid and carcharodontosaurid theropods from the Lower Cretaceous Elrhaz Formation of Niger. Acta Palaeontologica Polonica, 53 (1), 15-46.

Sereno, P.C., Dutheil, D.B., Iarochene, M., Larsson, H.C.E., Lyon, G.H., Magwene, P.M., Sidor, C.A., Varricchio, D.J. \& Wilson, J.A. 1996. Predatory dinosaurs from the Sahara and Late Cretaceous faunal differentiation. Science, 272, 986-991.

Smith, J.B. \& Lamanna, M.C. 2006. An abelisaurid from the Late Cretaceous of Egypt: implications for theropod biogeography. Naturwissenschaften, 93, 242-245.

Smith, J.B., Vann, D.R. \& Dodson, P. 2005. Dental morphology and variation on theropod dinosaurs: implications for the taxonomic identification of isolated teeth. The Anatomical Record, Part A: Discoveries in Molecular, Cellular, and Evolutionary Biology, 285A, 699-736.

Ten Cate, A.R. 1995. The experimental investigation of odontogenesis. International Journal of Developmental Biology, 39, 5-11.
Upchurch, P. \& Barrett, P.M. 2000. The evolution of sauropod feeding mechanisms. En: Evolution of Herbivory in Terrestrial Vertebrates: perspectives from the fósil record (ed. Sues, H.-D.). Cambridge University Press, Cambridge, 79-122.

Wilson, J.A. 2005. Redescription of the Mongolian sauropod Nemegtosaurus mongoliensis Nowinski (Dinosauria: Saurischia) and comments on Late Cretaceous sauropod diversity. Journal of Systematic Palaeontology, 3, 283318.

Woodward, A.S. 1896. On two Mesozoic crocodilians Notosuchus nov. gen. and Cynodontosuchus nov. gen. from the red sandstones of the Territory of Neuquén (Argentina). Anales del Museo de la Plata, Paleontología, 4, 1-20.

Woodward, A.S. 1901. On some extinct reptiles from Patagonia of the genera Miolania, Dinilysia and Genyodectes. Proccedings of the Royal Society of London, 1, 169-184. 
